\title{
STUDIES ON THE PIGMENTS OF MARINE ANIMALS -VIII. CAROTENOIDS OF SOME SHELLFISHES-
}

AUTHOR(S):

Nishibori, Kokichi

CITATION:

Nishibori, Kokichi. STUDIES ON THE PIGMENTS OF MARINE ANIMALS -VIII. CAROTENOIDS OF SOME SHELLFISHES-. PUBLICATIONS OF THE SETO MARINE BIOLOGICAL LABORATORY 1960, 8(2): 317-326

\section{ISSUE DATE:}

1960-12-20

URL:

http://hdl.handle.net/2433/174647

RIGHT: 


\title{
STUDIES ON THE PIGMENTS OF MARINE ANIMALS
}

VIII. CAROTENOIDS OF SOME SHELLFISHES

\author{
KOKICHI NISHIBORI
}

Notre Dame Seishin University, Okayama

With Plate $X X X V, 4$ Tables and 10 Text-figures

The foot, gonad and flesh of shellfishes, showing a red, orange and yellow colour, contain some carotenoid pigments. In the gonads and coats of Pectunculus glycymeris, glycymerin was found by FABRE and LEDERER (1934). LEDERER also discovered pectenoxanthin from the gonads of Pecten maximus (LEDERER, 1934). Mytyloxanthin and zeaxanthin were isolated from the orange coloured flesh of Mytilus californianus by SCHEer (1940). Red flesh of Tugali gigas contains astaxanthin and a new carotenoid (NISHIBORI, 1957).

Carotenoid pigments in the red-orange foot of Anadara broughtoni (Japanese name, Akagai), Anadara subcrenata (Japanese name, Sarubô), and in the redorange ovaries of Pecten yessoensis (Japanese name, Hotategai) have been examined and the principal carotenoids in each species were isolated in the crystalline form.

\section{Material and Method}

The colour, number and weight of the specimens used in this experiment are summarized in Table 1.

The extraction and separation method of the pigments is shown in Table 2.

Table 1. Colour, number and weight of the specimens.

\begin{tabular}{l|c|c|c}
\hline & Anadara broughtoni & Anadara subcrenata & Pecten yessoensis \\
\hline Organ & foot & foot & ovary \\
Colour & red-orange & red-orange & red-orange \\
Number & 64 & 1120 & 40 \\
Weight (g) & 400 & 2450 & 1500 \\
\hline
\end{tabular}

Publ. Seto Mar. Biol. Lab., VIII (2), 1960. (Article 24) 
Table 2. Extraction and separation method.

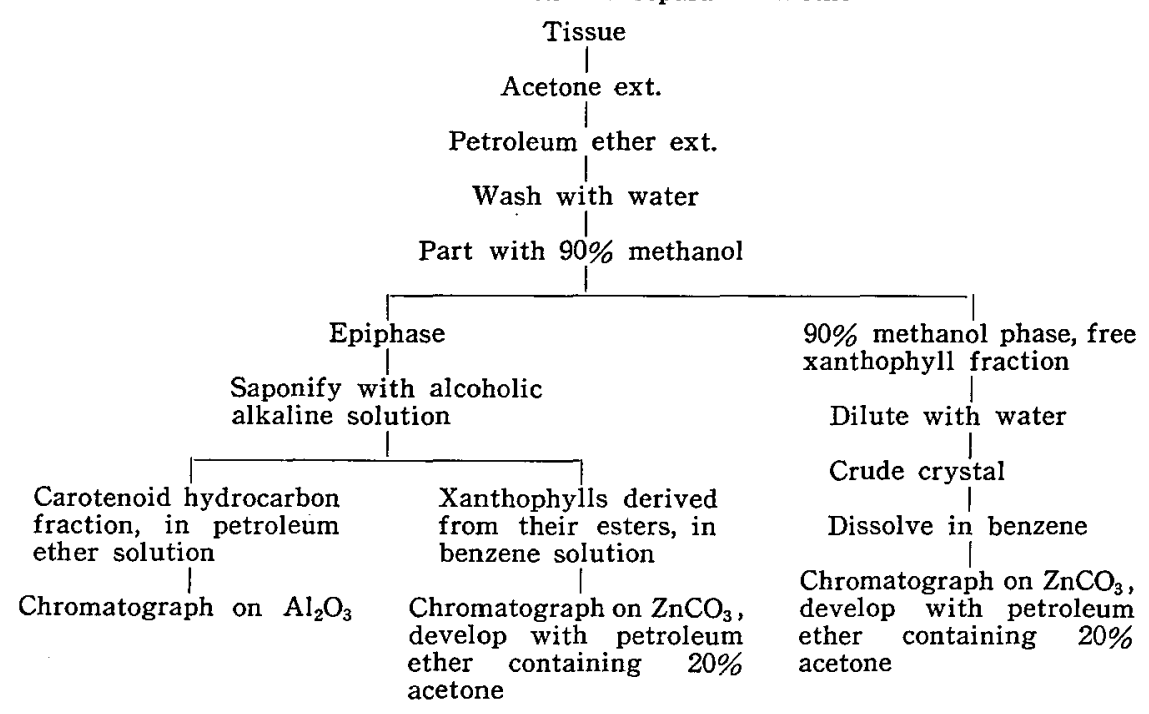

\section{Result}

\section{Anadara broughtoni}

An amount of $400 \mathrm{~g}$ of the fresh red-orange foot (64 specimens) collected at Fukugawa in Yamaguchi Pref. was used for extraction. Pigments were entirely extracted with acetone and most of the pigment transferred therefrom into the $90 \%$ methanol solution forming a deep red colour. The colour of the epiphasic phase was light yellow.

Free xanthophyllic fraction: Red-brown crude crystals $\left(\mathrm{mp} .172^{\circ}\right)$, which were separated when the $90 \%$ methanol solution was covered with a trace of petroleum ether and diluted with water, were dissolved in benzene and chromatographed upon the column of zinc carbonate and developed with petroleum ether containing 20\% acetone. Three zones were obtained from the top of the column. The upper zone $f(I)$ was a light red-orange in colour and the intermediate zone $f(I I)$ was red-organge in colour. The pigment in $f(I I)$ was eluted with acetic acidacetone mixture and the red substance was obtained. CARR-PRICE reaction $(+)$ blue, conc. $\mathrm{H}_{2} \mathrm{SO}_{4}$ reaction $(+$ ) blue, absorption maximum was at $506 \mathrm{~m} \mu$ in carbon disulphide (Fig. 1). This hypophasic carotenoid has a ketonic property. From the lower deep yellow zone $\mathrm{f}(\mathrm{III})$, containing the principal pigment of the specimen, orange-yellow needle-like crystals were obtained and recrystallized from ethanol-water. The yield was about $0.5 \mathrm{mg}$ and $\mathrm{mp}$. was $181^{\circ}$ (Plate XXXV, fig. 1 ). CARR-PRICE and conc. $\mathrm{H}_{2} \mathrm{SO}_{4}$ reactions were positive, blue and absorption maxima were at $\sim 450,480,512 \mathrm{~m} \mu$ in carbon disulphide, $\sim 435,463,492 \mathrm{~m} \mu$ in chloroform and $\sim 435,464,494 \mathrm{~m} \mu$ in benzene (Fig. 1, Fig. 2). It has a hypophasic property 
by partition between petroleum ether and $90 \%$ methanol but does not possess a ketonic property.

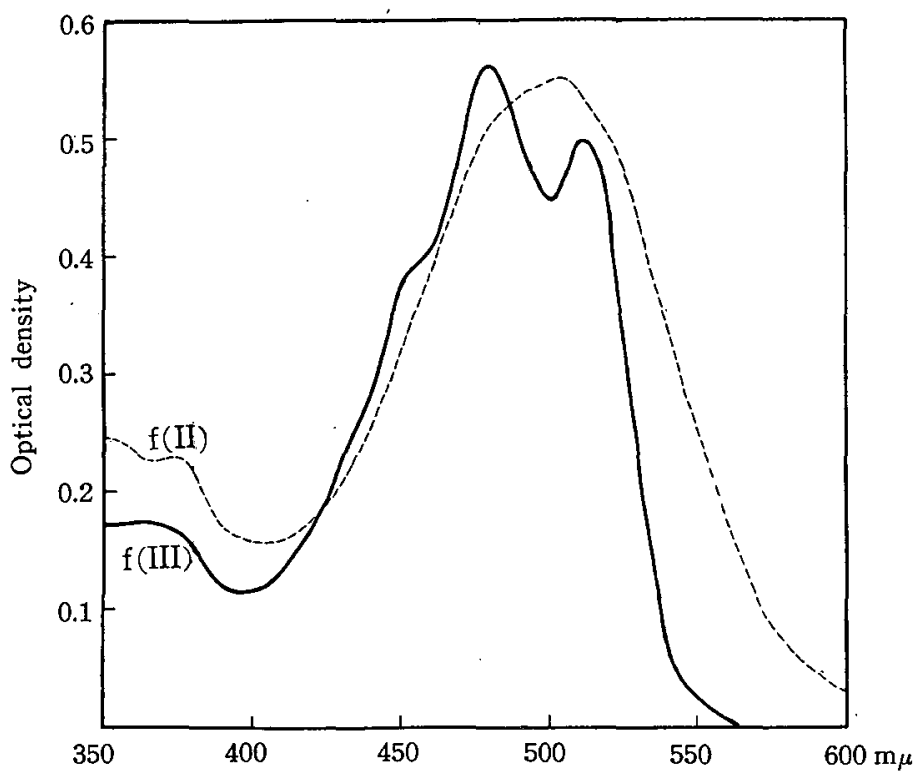

Fig. 1. Absorption spectra of free xanthophyll fraction of Anadara broughtoni; free xanthophyll of $\mathrm{f}(\mathrm{II})$ and $f(I I I)\left(m p .181^{\circ}\right.$ ) in carbon disulphide.

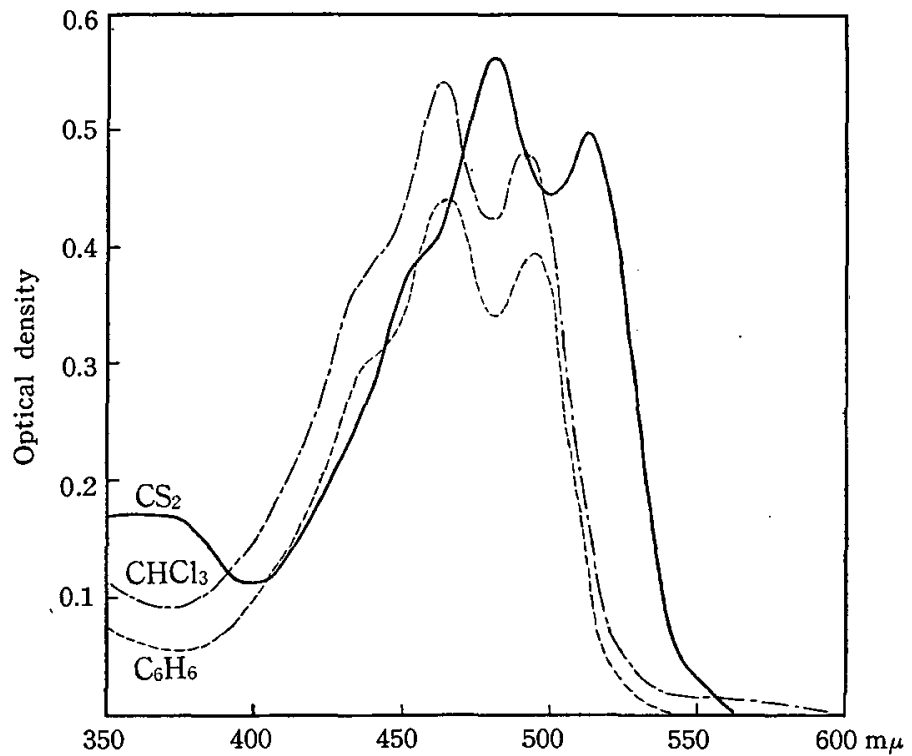

Fig. 2. Absorption spectra of the principal crystalline carotenoid, mp. $181^{\circ}$ in Anadara broughtoni (in various solvents). 
Esterified xanthophyll fraction: The carotenoid of this fraction in benzene was chromatographed upon the column of zinc carbonate and developed with petroleum ether containing $20 \%$ acetone. Two zones appeared; the upper red zone $f(I)$ contains a red carotenoid which is eluted with acetic acid-acetone. CARR-PRICE reaction, violet-blue, conc. $\mathrm{H}_{2} \mathrm{SO}_{4}$ reaction, blue, absorption maxima are at $\sim 445,478,505 \mathrm{~m} \mu$ in carbon disulphide (Fig. 3 ). It possesses a hypophasic property. The pigment which was eluted from the lower orange zone $f(I I)$, showed the absorption maxima at $\sim 445,481,510 \mathrm{~m} \mu$ in carbon disulphide (Fig. 3 ). CARR-PRICE and conc. $\mathrm{H}_{2} \mathrm{SO}_{4}$ reactions $(+)$ blue.

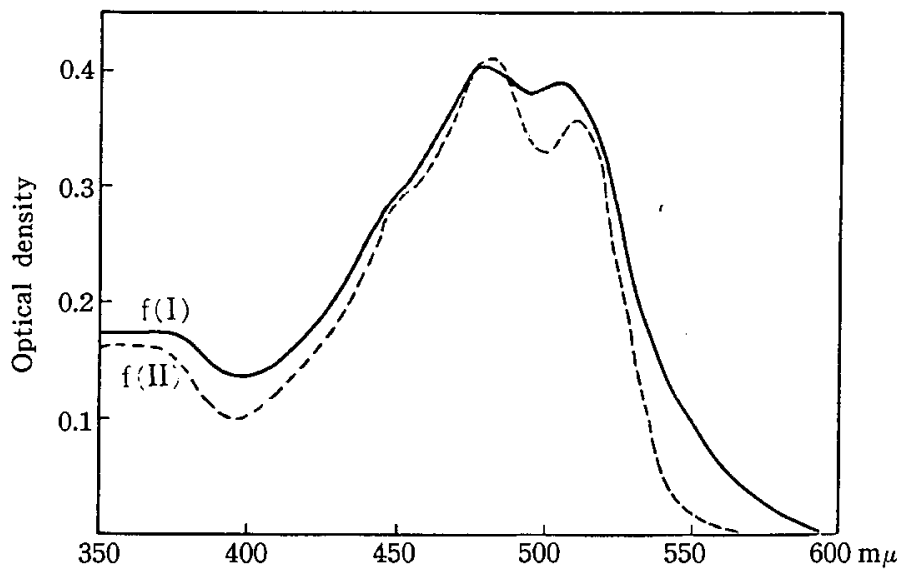

Fig. 3. Absorption spectra of $f(I)$ and $f(I I)$ derived from their esters in Anadara broughtoni (in carbon disulphide).

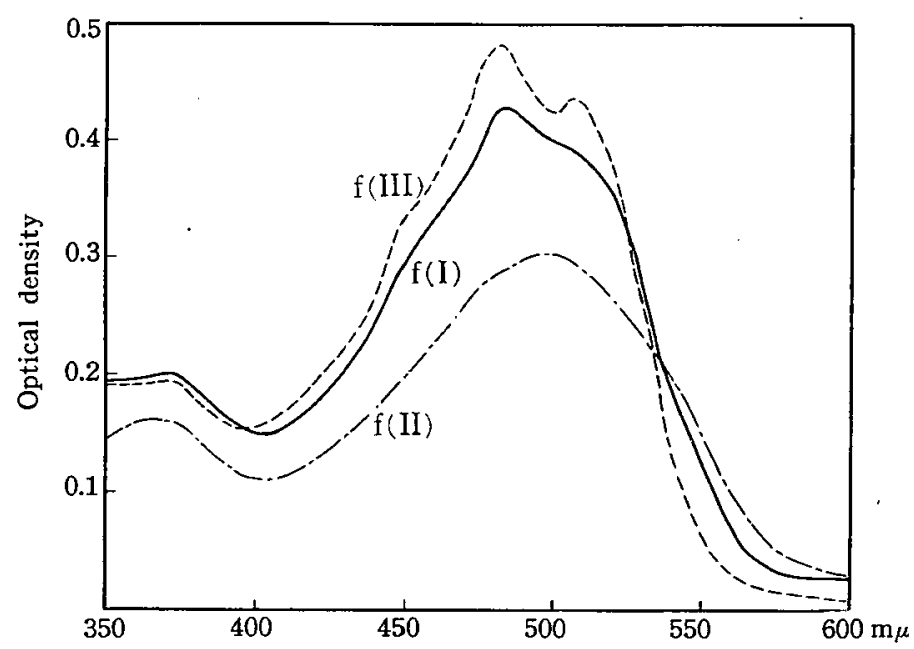

Fig. 4. Absorption spectra of carotenoid hydrocarbon fraction, f(I), f(II) and $\mathrm{f}(\mathrm{III})$ in Anadara broughtoni (in carbon disulphide). 
Carotenoid hydrocarbon fraction: Petroleum ether solution of the pigments of this fraction were chromatographed upon alumina (Merck) and developed with petroleum ether containing $1 \%$ acetone. Three zones, yellow zone $f(I)$, orangeyellow zone $f(I I)$ and bright yellow zone $f(I I I)$, were obtained from the top of the column. Carotenoids of each zone were eluted with acetone. The absorption maxima in carbon disulphide were as follows; (Fig. 4). $\mathrm{f}(\mathrm{I}), \sim 445,485, \sim 515 \mathrm{~m} \mu$, f(II), $495 \sim 500 \mathrm{~m} \mu, \mathrm{f}(\mathrm{III}), \sim 450,481,507 \mathrm{~m} \mu$.

\section{Anadara subcrenata}

Red-orange foot, $2450 \mathrm{~g}$ in weight, were used for extraction. The specimens were collected at Sami in Okayama Pref.

Free xanthophyllic fraction: The greatest amount of the pigment was found in this fraction. Red-brown crude crystals were precipitated at the boundary between the petroleum ether and dilute $90 \%$ methanol.

The yield was about $1.5 \mathrm{mg}, \mathrm{mp} .168^{\circ}$. When the crystals were dissolved in benzene, chromatographed upon the column of zinc carbonate, and developed with petroleum ether solution containing acetone (20\%), three zones appeared. The pigment in the upper red-orange zone $f(I)$, was eluted with acetic acid-acetone from which the red amorphous substance was separated. The properties of this pigment were as follows; CARR-PRICE reaction, $(+)$ blue, conc. $\mathrm{H}_{2} \mathrm{SO}_{4}$ reaction, (+) blue, absorption maximum, $500 \sim 505 \mathrm{~m} \mu$ in carbon disulphide, (Fig. 5), $475 \sim 490 \mathrm{~m} \mu$ in chloroform. It has a ketonic property. The pigment in the intermediate zone $\mathrm{f}(\mathrm{II})$, having an orange colour, was also eluted with acetic acidacetone and was crystallized in a red needle form from the hot ethanol, mp. 162

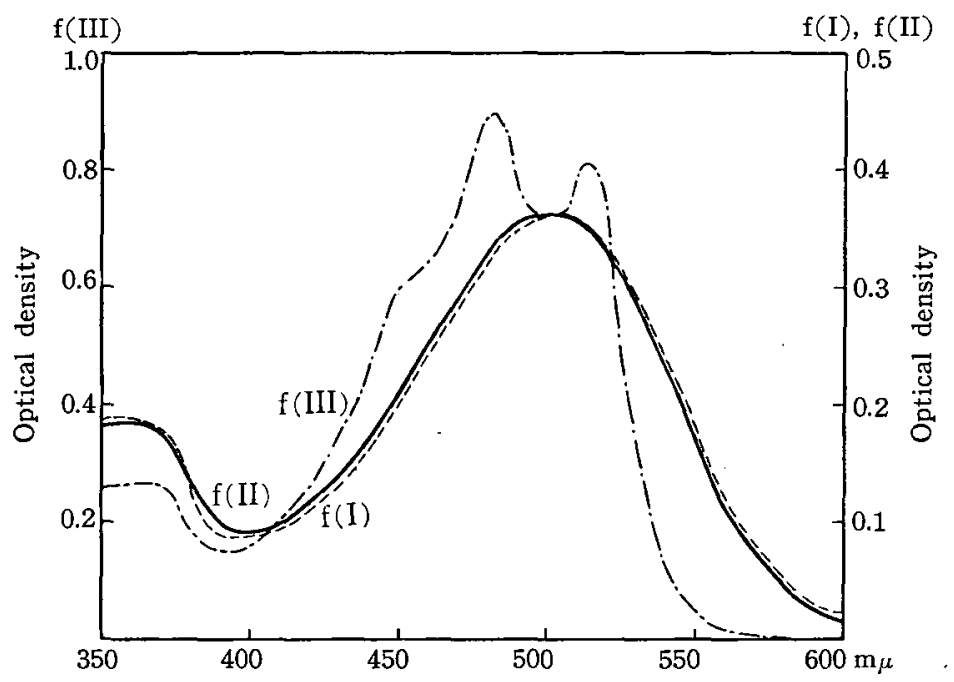

Fig. 5. Absorption spectra of free xanthophyll fraction, f(I), $\mathrm{f}(\mathrm{II}) \mathrm{mp.} 162-3^{\circ}$ and $\mathrm{f}(\mathrm{III}) \mathrm{mp} .183-4^{\circ}$ in Anadara subcrenata (in carbon disulphide). 
$3^{\circ}$. It has a ketonic property showing the absorption maximum at $498 \sim 508 \mathrm{~m} \mu$ in carbon disulphide (Fig. 5) and $480 \sim 490 \mathrm{~m} \mu$ in chloroform. CARR-PRICE and conc. $\mathrm{H}_{2} \mathrm{SO}_{4}$ reactions $(t)$ blue. The lower main zone $\mathrm{f}(\mathrm{III})$, having a deep yellow colour, contains the principal carotenoid of this specimen. Orange yellow prisms were obtained and recrystallized from hot ethanol (Plate XXXV, fig. 2), mp. $183 \sim 4^{\circ}$. Absorption maxima were at $\sim 450,482,512 \mathrm{~m} \mu$ in carbon disulphide, $\sim 435,462,492 \mathrm{~m} \mu$ in chloroform and $\sim 435,464,494 \mathrm{~m} \mu$ in benzene (Fig. 5, Fig. 6). It showed entirely a hypophasic property. CARR-PRICE and conc. $\mathrm{H}_{2} \mathrm{SO}_{4}$ reactions were positive, blue.

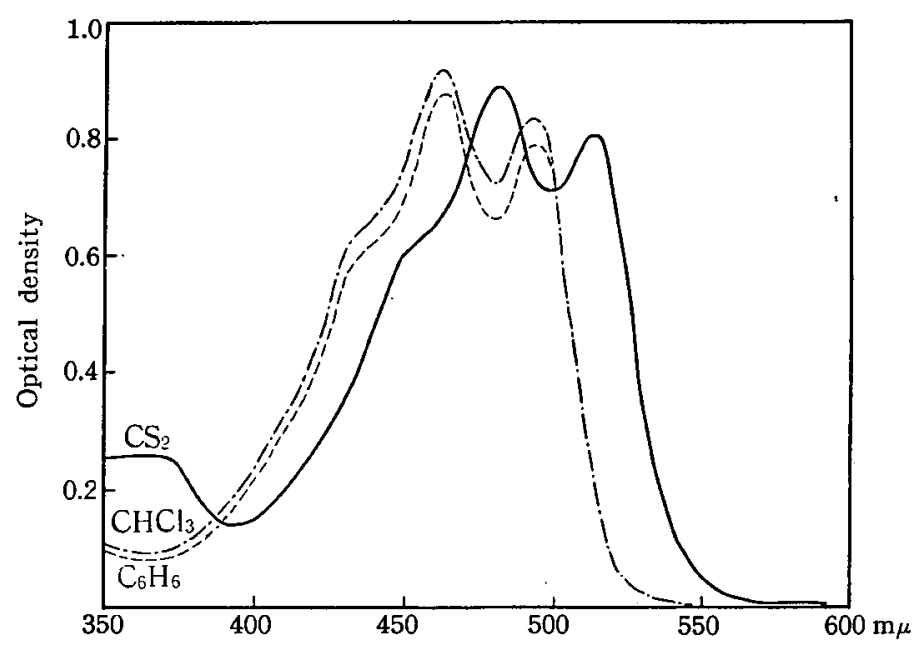

Fig. 6. Absorption spectra of the principal crystalline carotenoid mp. $183-4^{\circ}$ in Anadara subcrenata (in various solvents).

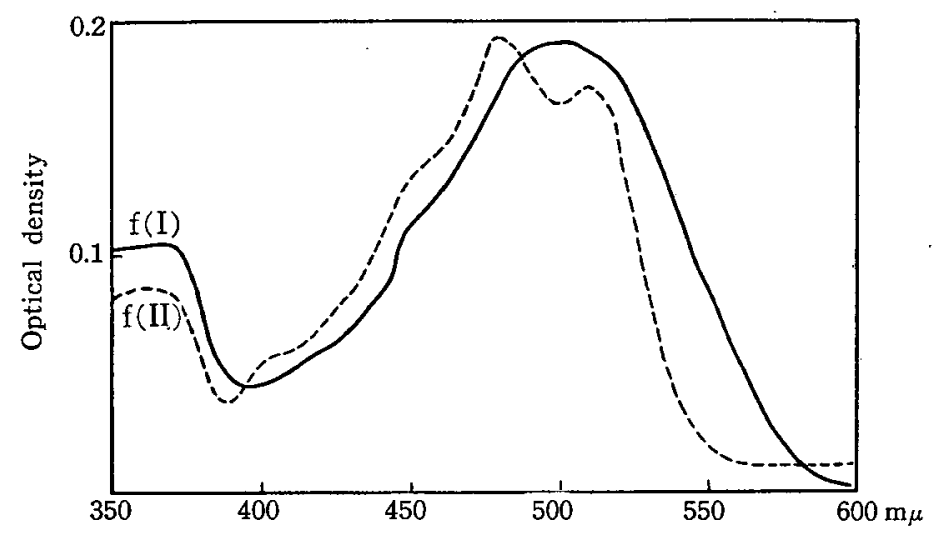

Fig. 7. Absorption spectra of $f(I)$ and $f(I I)$ derived from their esters in Anadara subcrenata (in carbon disulphide). 
Esterified xanthophyll fraction: After chromatographic adsorption upon zinc carbonate from benzene solution and development with petroleum ether containing acetone (20\%), two fractions were obtained. From the upper red zone $f(I)$, an amorphous red substance was eluted with acetic acid-acetone which showed the absorption maxima at $\sim 445, \sim 502 \mathrm{~m} \mu$ in carbon disulphide (Fig. 7). It has a ketonic property. CARR-PRICE reaction $(t)$ blue. The pigment in the lower orange zone $\mathrm{f}(\mathrm{II})$, was eluted with neutral acetone. It possessed a hypophasic property and the absorption maxima at $\sim 450,478,508 \mathrm{~m} \mu$ in carbon disulphide (Fig. 7). CARR-PRICE reaction, (+) blue.

Carotenoid hydrocarbon fraction: Three zones were obtained when the petroleum ether solution of the pigment in this fraction was chromatographed upon alumina and developed with petroleum ether containing $4 \%$ acetone. Absorption maxima of the pigment of each zone in carbon disulphide were as follows (Fig. 8).

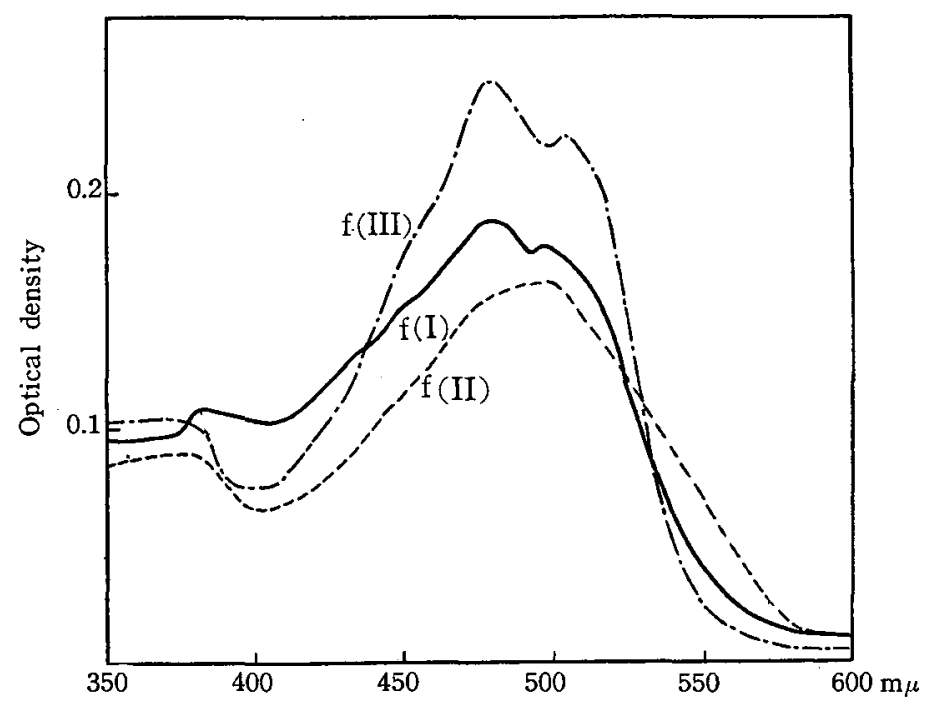

Fig. 8. Absorption spectra of carotenoid hydrocarbon fraction, $\mathrm{f}(\mathrm{I}), \mathrm{f}(\mathrm{II})$ and $\mathrm{f}(\mathrm{III})$ in Anadara sub. crenata (in carbon disulphide).

Light orange zone $f(I)$ (the upper)

Light pink zone $f($ II) (the intermediate)

Deep orange zone $f($ III) (the lower)
( $\sim 435),(\sim 450), 480,496 \mathrm{~m} \mu$

$\sim 475, \sim 500 \mathrm{~m} \mu$

$\sim 450,480,502 \sim 506 \mathrm{~m} \mu$

CARR-PRICE reaction of each carotenoid was blue.

\section{Pecten yessoensis}

An amount of $1500 \mathrm{~g}$ of ovaries from 40 specimens which were obtained at a fish market in Sapporo City was used for extraction. The red-orange pigment in acetone extract was transferred therefrom into petroleum ether and then into $90 \%$ methanol solution. When the $90 \%$ methanol solution of the pigment was 
diluted with water after the addition of a layer of petroleum ether, the orange-red crystals appeared at the boundary of the two layers. After washing with petroleum ether, the crystals were recrystallized from aqueous pyridine into orange prisms which melted at $181 \sim 182^{\circ}$ (Plate XXXV, fig. $3)$. The yield was about $1.5 \mathrm{mg}$. It forms a single zone when it is chromatographed upon the column of calcium carbonate from petroleum ether solution containing a trace of pyridine and developed with petroleum etheracetone mixture. Absorption maxima were at $\sim 434,465,496 \mathrm{~m} \mu$ in benzene

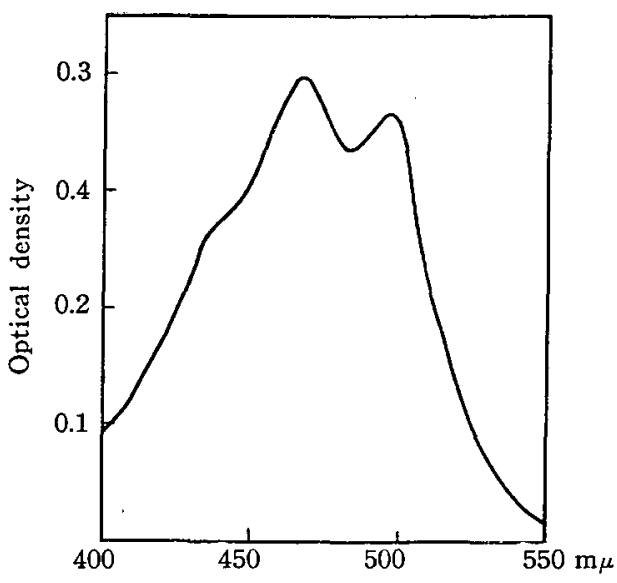

Fig. 9. Absorption spectrum of pectenoxanthin in the ovaries of Pecten yessoensis (in benzene).

(Fig. 9). It possessed a hypophasic

property, CARR-PRICE reaction $(+)$, blue and conc. $\mathrm{H}_{2} \mathrm{SO}_{4}$ reaction $(+)$, blue.

\section{Discussion}

Carotenoid in foot: The principal pigment in the foot of two species of the shellfishes, Anadara broughtoni and Anadara subcrenata is free xanthophyllic carotenoid and the amount of esterified xanthophyll and carotenoid hydrocarbon is poor. The absorption maxima of the carotenoids obtained are summarized in Table 3.

Table 3. Absorption maxima of carotenoids in the foot of shellishes Anadara broughtoni and Anadara subcrenata (in carbon disulphide).

\begin{tabular}{|c|c|c|c|c|c|}
\hline & & \multicolumn{2}{|c|}{ Anadara broughtoni } & \multicolumn{2}{|c|}{ Anadara subcrenata } \\
\hline & & $\operatorname{mp} .\left({ }^{\circ} \mathrm{C}\right)$ & absorp. $\max .(\mathrm{m} \mu)$ & $\mathrm{mp} \cdot\left({ }^{\circ} \mathrm{C}\right)$ & absorp. $\max .(\mathrm{m} \mu)$ \\
\hline \multirow[t]{2}{*}{$\begin{array}{l}\text { Free xanthophyllic } \\
\text { fraction }\end{array}$} & $\begin{array}{l}f(I) \\
f(I I)\end{array}$ & 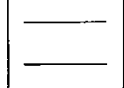 & 506 & $\mid-162 \sim 3$ & $\begin{array}{l}500 \sim 505 \\
498 \sim 508\end{array}$ \\
\hline & $\mathrm{f}(\mathrm{III})$ & 181 & $\sim 450,480$ & $183 \sim 4$ & $\sim 450, \quad 482,512$ \\
\hline $\begin{array}{l}\text { Esterified xantho- } \\
\text { phyll fraction }\end{array}$ & $\begin{array}{l}\mathbf{f}(\mathrm{I}) \\
\mathbf{f}(\mathrm{II})\end{array}$ & & $\begin{array}{ll}\sim 445,478, & 505 \\
\sim 445,481, & 510\end{array}$ & & $\begin{array}{l}\sim 445, \sim 520 \\
\sim 450, \quad 478,508\end{array}$ \\
\hline $\begin{array}{l}\text { Carotenoid hydro- } \\
\text { carbon fraction }\end{array}$ & $\begin{array}{l}\mathbf{f}(\mathrm{I}) \\
\mathbf{f}(\mathrm{II}) \\
\mathbf{f}(\mathrm{III})\end{array}$ & 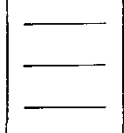 & $\begin{array}{l}\sim 445,485, \sim 515 \\
495 \sim 500 \\
\sim 450,481, \quad 507\end{array}$ & - & $\begin{array}{l}\sim 435, \sim 450,480,496 \\
\sim 475, \sim 500 \\
\sim 450, \quad 480, \quad 502 \sim 6\end{array}$ \\
\hline
\end{tabular}


The properties of the principal crystalline carotenoid in the foot of two species of the sea shell are very similar to each other (Table 4).

Table 4. Properties of the principal carotenoid in the foot of Anadara broughtoni and Anadara subcrenata.

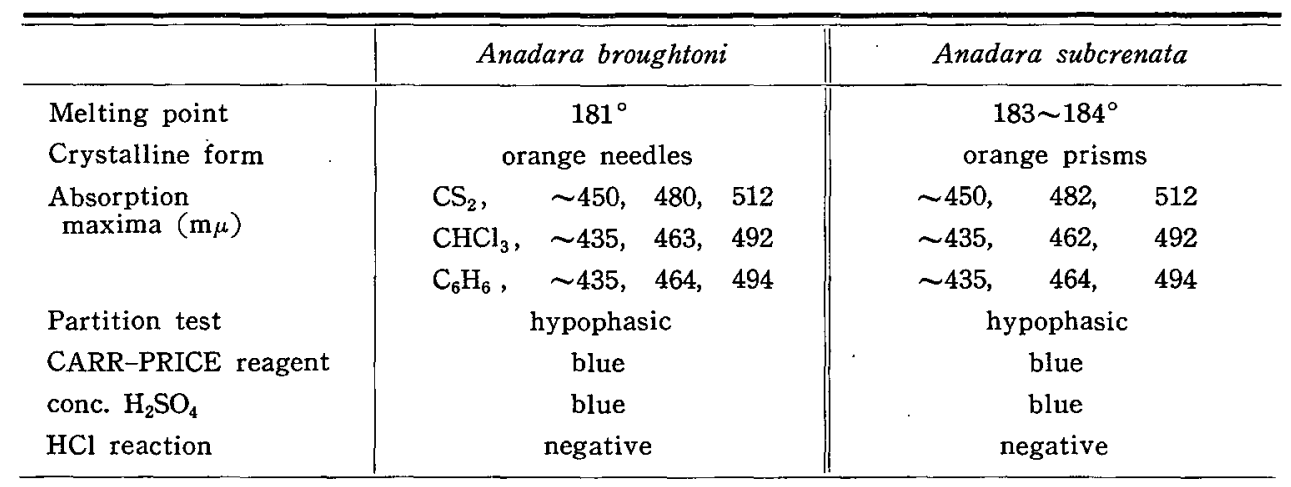

When the mixed benzene solution of the two crystals, mp. $181^{\circ}$ of $A$. broughtoni and mp. $183 \sim 4^{\circ}$ of $A$. subcrenata, was chromatographed upon the column of zinc carbonate and developed with petroleum ether containing $20 \%$ acetone, a single zone appeared. The absorption curve of both two crystals was fairly agreeable (Fig. 10). These results show that the two crystalline carotenoids are identical

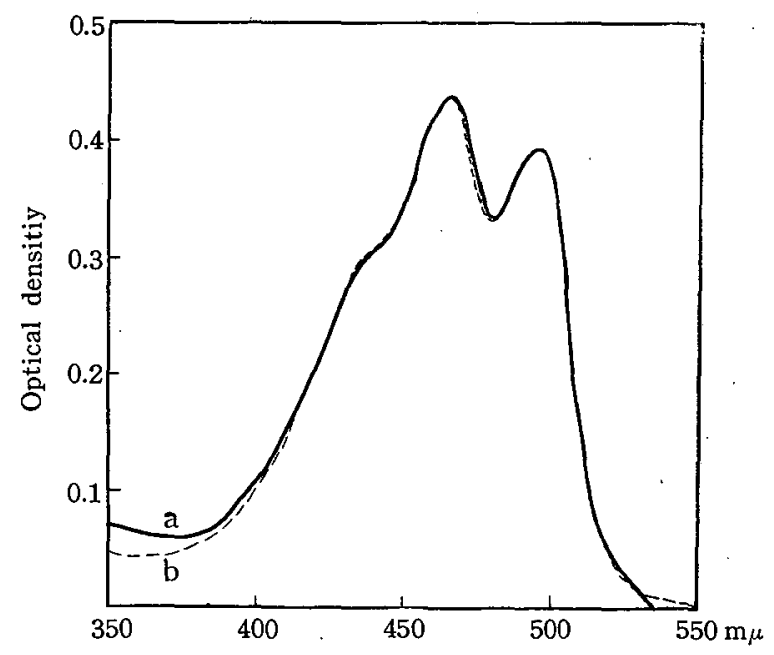

Fig. 10. Absorption spectra of the principal crystalline carotenoid in the foot of sea shell;

(a) $\cdots$ mp. $181^{\circ}$ carotenid in Anadara broughtoni

(b) $\cdots$ mp. $183 \sim 184^{\circ}$ carotenoid in Anadara subcrenata

(in benzene). 
with each other. This carotenoid seems to be almost identical with pectenoxanthin, but unfortunately direct comparison with pectenoxanthin did not carry out because of the lack of the crystals.

Carotenoid in ovary: The properties of the crystalline carotenoid in the orange coloured ovaries of Pecten yessoensis, such as mp., absorption maxima, indicate that this carotenoid is identical with pectenoxanthin ( $\mathrm{mp} .182^{\circ}$, absorption maxima, 434, $464,496 \mathrm{~m} \mu$ in benzene).

\section{Summary}

Carotenoids in the red-orange coloured foot of the shellfishes, Anadara broughtoni and Anadara subcrenata, and in the red-orange ovaries of Pecten yessoensis have been examined and the principal crystalline carotenoids were isolated in each species. The principal carotenoids of $A$. broughtoni (mp. $181^{\circ}$ ) and $A$. subcrenata (mp. $183 \sim 184^{\circ}$ ) are identical with each other and seem to be almost identical with pectenoxanthin. The ovary carotenoid of Pecten yessoensis (mp. 181 182 ${ }^{\circ}$ ) is identical with pectenoxanthin.

\section{Acknowledgment}

The author wishes to express his deep gratitude to Professor Y. Nakamura and Professor T. SAITo of Hokkaido University, for their encouragement and guidance. The author is also grateful to Dr. H. Utinom of Seto Marine Biological Laboratory of Kyoto University who gave the opportunity for publication.

\section{REFERENCES}

Fabre, R. et Lederer, E. 1934. Contributions à l'étude des lipochromes des animaux. Bull, Soc. Chim. biol., Paris, 16: 105.

Lederer, E; 1934. Pigment found in the mollusk Pecten maximus. Compt. rend. Soc. biol., 116 : 150-153.

NishibORI, K. 1957. Studies on pigments of marine animals V. Carotenoids from the red muscle and the viscera of giant shield shell, Tugali gigas. Bull. Jap. Soc. Sci. Fish., 22: 715-717.

SCHEER, B. T. 1940. Some features of the metabolism of the carotenoid pigments of the California sea mussel (Mytilus californianus). J. Biol. Chem., 136 : 275-99.

\section{EXPLANATION OF PLATE XXXV}

Fig. 1. Principal crystalline carotenoid, $\mathrm{mp} .181^{\circ}$, isolated from the foot of Anadara broughtoni.

Fig. 2. Principal crystalline carotenoid, mp. $183 \sim 184^{\circ}$, isolated from the foot of Anadara subcrenata.

Fig. 3. Pectenoxanthin, mp. $181^{\circ}$, isolated from the ovaries of Pecten yessoensis. 
Publ. Seto Mar. Biol. Lab., VIII, 2 (1960)

PLATE XXXV
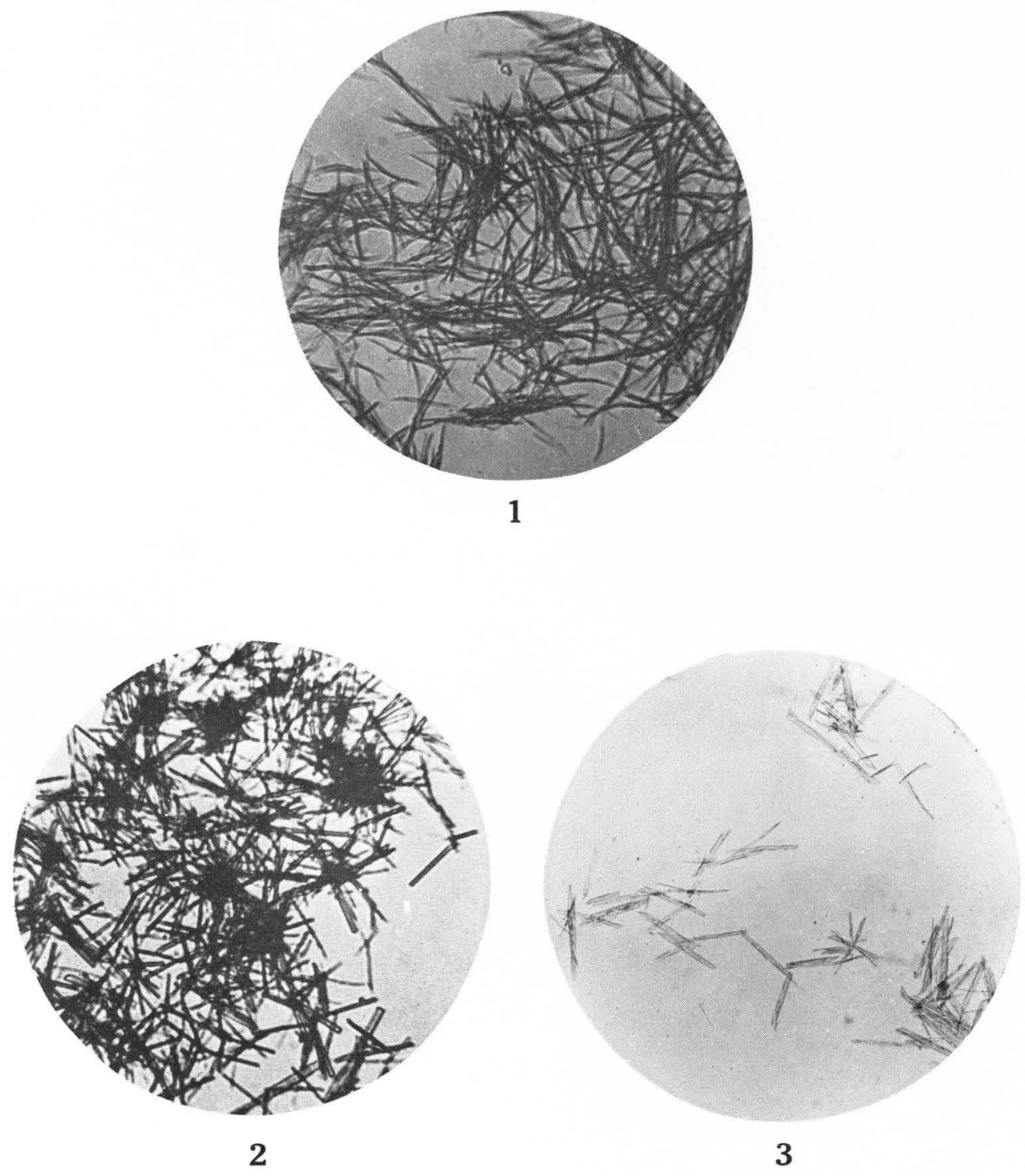

K. Nishibori: Studies on the Pigments of Marine Animals, ViII. 